\title{
Relative Distance-An Error Measure in Round-Off Error Analysis
}

\author{
By Abraham Ziv*
}

\begin{abstract}
Olver (SIAM J. Numer. Anal., v. 15, 1978, pp. 368-393) suggested relative precision as an attractive substitute for relative error in round-off error analysis. He remarked that in certain respects the error measure $d(\bar{x}, x)=\min \{\alpha \mid 1-\alpha \leqslant x / \bar{x} \leqslant 1 /(1-\alpha)\}, \bar{x} \neq 0$, $x / \bar{x}>0$ is even more favorable, through it seems to be inferior because of two drawbacks which are not shared by relative precision: (i) the inequality $d\left(\bar{x}^{k}, x^{k}\right) \leqslant|k| d(\bar{x}, x)$ is not true for $0<|k|<1$. (ii) $d(\bar{x}, x)$ is not defined for complex $\bar{x}, x$. In this paper the definition of $d(\cdot, \cdot)$ is replaced by $d(\bar{x}, x)=|\bar{x}-x| / \max \{|\bar{x}|,|x|\}$. This definition is equivalent to the first in case $\bar{x} \neq 0, x / \bar{x}>0$, and is free of (ii). The inequality $d\left(\bar{x}^{k}, x^{k}\right) \leqslant|k| d(\bar{x}, x)$ is replaced by the more universally valid inequality $d\left(\bar{x}^{k}, x^{k}\right) \leqslant|k| d(\bar{x}, x) /(1-\delta), \delta=$ $\max \{d(\bar{x}, x),|k| d(\bar{x}, x)\}$. The favorable properties of $d(\cdot, \cdot)$ are preserved in the complex case. Moreover, its definition may be generalized to linear normed spaces by $d(\bar{x}, x)=$ $\|\bar{x}-x\| / \max \{\|\bar{x}\|,\|x\|\}$. Its properties in such spaces raise the possibility that with further investigation it might become the basis for error analysis in some vector, matrix, and function spaces.
\end{abstract}

1. Introduction. Let $\bar{x}$ be a real number which is an approximation of another real number $x$. The relative error

$$
\rho(\bar{x}, x)=(\bar{x}-x) / x \quad(x \neq 0),
$$

is often used to measure the derivation of $\bar{x}$ from $x$. This definition is very simple and its meaning is intuitively clear. It has however certain annoying drawbacks which have to do primarily with the elegance of error analysis, the simplicity of error bounds and the ease with which they are derived. To be more specific:

(a) $|\rho(\bar{x}, x)|$ is not a metric, and often $|\rho(\bar{x}, x)| \neq|\rho(x, \bar{x})|,|\rho(x, z)| \neq$ $|\rho(x, y)|+|\rho(y, z)|$.

(b) Let $\bar{x}_{i}$ be an approximation of $x_{i}$, and denote $\rho_{i}=\rho\left(\bar{x}_{i}, x_{i}\right)$. Let $y=\Pi_{i} x_{i}^{r_{i}}$, where $r_{i}$ are integers. Then $\bar{y}=\prod_{i} \bar{x}_{i}^{r_{i}}$ is an approximation of $y$. To the first order, in $\max \left|\rho_{i}\right|, \rho(\bar{y}, y)$ is equal to $\Sigma_{i} r_{i} \rho_{i}$, so some authors take $\Sigma_{i}\left|r_{i} \rho_{i}\right|$ as an approximate bound of $\rho(\bar{y}, y)$; see, e.g., [1]. Unfortunately, though, this is not a true bound.

In view of this, one is tempted to try to replace the familiar concept of relative error by something else. Olver [3] suggests the so-called 'relative precision' as a substitute. He denotes

$$
a \simeq \tilde{a} ; \quad \operatorname{rp}(\alpha)
$$

Received November 17, 1980; revised November 17, 1981.

1980 Mathematics Subject Classification. Primary 65G05.

Key words and phrases. Relative error, round-off error analysis, metric.

*The author is with the IBM Israel Scientific Center, Technion City, Haifa 32000, Israel. The paper was revised while visiting IBM Thomas J. Watson Research Center in Yorktown Heights, New York. 
whenever $a / \tilde{a}=e^{u}$, where $|u| \leqslant \alpha$. In the spirit of (1) one might define

$$
\operatorname{rp}(\bar{x}, x)=\log (\bar{x} / x) \quad(x \neq 0, \bar{x} / x>0),
$$

so (2) becomes equivalent to $|\operatorname{rp}(a, \tilde{a})| \leqslant \alpha$. Obviously, $\operatorname{rp}(\bar{x}, x)=\rho(\bar{x}, x)+$ $O\left(\rho^{2}(\bar{x}, x)\right)$, and therefore relative precision does not differ much from relative error. It is, however, free from the annoying (a) and (b) and has some other convenient properties.

Another substitute mentioned in [3] is what shall be referred to later as 'relative distance':

$$
d(\bar{x}, x)=\min \{\alpha \mid 1-\alpha \leqslant x / \bar{x} \leqslant 1 /(1-\alpha)\} \quad(\bar{x} \neq 0, x / \bar{x}>0) .
$$

Obviously, $d(\bar{x}, x)=|\rho(\bar{x}, x)|+O\left(\rho^{2}(\bar{x}, x)\right)$, and it is also free of (a), (b).

Relative distance and relative precision are quite similar in their properties which have to do with error analysis. In spite of the simplicity of definition and of error bounds with relative distance, compared to relative precision, and in spite of the fact that relative distance corresponds more closely to relative error (relative error may be defined also by $\rho(\bar{x}, x)=\min \{\varepsilon \mid 1-\varepsilon \leqslant \bar{x} / x \leqslant 1+\varepsilon\}, x \neq 0$ ), relative precision was preferred in [3] because of two reasons:

(i) For every real $k$

$$
a \simeq \tilde{a} ; \quad \operatorname{rp}(\alpha) \Rightarrow a^{k} \simeq \tilde{a}^{k} ; \quad \operatorname{rp}(|k| \alpha),
$$

whereas the necessary condition $|k| \geqslant 1$ restricts the analogous property of relative distance.

(ii) The definition of relative precision carries over to complex numbers with all its important properties preserved. It is not clear, on the other hand, how to generalize (4) to complex $\bar{x}, x$.

In what follows, the definition of relative distance is generalized in a way that eliminates (ii). It is shown that the property mentioned in (i) has a reasonable substitute, which is valid for relative distance without the restriction $|k| \geqslant 1$. The most interesting properties of relative precision are shown to hold for the generalized form of relative distance too-not only for real numbers but also for complex numbers. Moreover, relative distance is generalized to linear normed spaces. Its properties in such spaces raise the possibility that, with further investigation, it might become the basis for error analysis in some vector, matrix, and function spaces.

2. Relative Distance in the Spaces of Real or Complex Numbers. The following definition of relative distance is meaningful for both real and complex $\bar{x}, x$ :

$$
d(\bar{x}, x)=|\bar{x}-x| / \max \{|\bar{x}|,|x|\}, \quad d(0,0)=0 .
$$

One should notice its simplicity, compared to the definition of relative precision, particularly in cases where $\bar{x} / x$ is not real positive. It is not difficult to show that this is equivalent to (4) whenever (4) is valid.

The following favorable properties of relative precision, with real numbers, were mentioned in [3]. They carry over to complex numbers with very little or no change:

(I) Symmetry: $a \simeq \tilde{a} ; \operatorname{rp}(\alpha) \Rightarrow \tilde{a} \simeq a ; \operatorname{rp}(\alpha)$.

(II) Inclusion: $a \simeq \tilde{a} ; \operatorname{rp}(\alpha), \delta \geqslant \alpha \Rightarrow a \simeq \tilde{a}$; $\operatorname{rp}(\delta)$.

(III) $a \simeq \tilde{a} ; \operatorname{rp}(\alpha) \Rightarrow k a \simeq k \tilde{a} ; \operatorname{rp}(\alpha)(k \neq 0, k$ real $)$. 
(IV) $a \simeq \tilde{a} ; \operatorname{rp}(\alpha) \Rightarrow a^{k} \simeq \tilde{a}^{k} ; \operatorname{rp}(|k| \alpha)(\tilde{a}, a$ real positive, $k$ real $)$.

(V) $a \simeq \tilde{a} ; \operatorname{rp}(\alpha), b \simeq \tilde{b} ; \operatorname{rp}(\beta) \Rightarrow a b \simeq \tilde{a} \tilde{b}, a / b \simeq \tilde{a} / \tilde{b} ; \operatorname{rp}(\alpha+\beta)$.

(VI) Triangle inequality: $a \simeq \tilde{a} ; \operatorname{rp}(\alpha), \tilde{a} \simeq \tilde{a} ; \operatorname{rp}(\beta) \Rightarrow a \simeq \tilde{a} ; \operatorname{rp}(\alpha+\beta)$.

An error analysis based on relative distance will obviously satisfy analogues of (I), (II), (III). The following theorem is related to (VI):

TheOREM 1. $d(\cdot, \cdot)$, defined by (5), is a metric on the space of complex numbers.

Proof. This theorem is an immediate corollary of the more general Theorem $1^{\circ}$; see next section. Therefore, we omit its proof here and refer to the next section.

Conditions (IV) and (V) were not discussed yet. As for (V) one gets for real or complex $x, \bar{x}, y, \bar{y}$ :

$$
\begin{aligned}
d(\bar{x} \bar{y}, x y) & \leqslant d(\bar{x} \bar{y}, x \bar{y})+d(x \bar{y}, x y)=d(\bar{x}, x)+d(\bar{y}, y), \\
d(\bar{x} / \bar{y}, x / y) & =d(\bar{x} \cdot 1 / \bar{y}, x \cdot 1 / y) \leqslant d(\bar{x}, x)+d(1 / \bar{y}, 1 / y) \\
& =d(\bar{x}, x)+d(\bar{y}, y) .
\end{aligned}
$$

From this an analogue of (IV) follows immediately for real or complex $\bar{x}, x$ and integral $k$ :

$$
d\left(\bar{x}^{k}, x^{k}\right) \leqslant|k| d(\bar{x}, x) \quad(\bar{x}, x \text { real or complex, } k \text { integer }) .
$$

It should be observed that nothing more general than (6) is necessary in error analysis of computations with real or complex numbers, provided only the four arithmetic operations are involved. Actually (6) is true also for values of $k$ other than integral but not for all values of $k$ (take, for example, real positive $\bar{x}, x$ and $k$ real, $0<|k|<1)$. The following theorem, however, seems to provide a reasonable substitute which is more universal.

THEOREM 2. Let $\bar{x}, x, k$ be complex numbers that satisfy $\max \{d(\bar{x}, x),|k| d(\bar{x}, x)\}$ $=\delta<1, \bar{x} x \neq 0$. If $x^{k}=\exp (k \log x), \bar{x}^{k}=\exp (k \log \bar{x})$ where the $\log$ branches are such that $-3 \pi / 2 \leqslant \operatorname{Im}\{\log \bar{x}-\log x\} \leqslant 3 \pi / 2$ (actually $\delta<1 \Rightarrow-\pi / 2<$ $\operatorname{Im}\{\log \bar{x}-\log x\}<\pi / 2)$, then

$$
d\left(\bar{x}^{k}, x^{k}\right) \leqslant|k| d(\bar{x}, x) /(1-\delta) .
$$

Note that $|k| d(\bar{x}, x) /(1-\delta)=|k| d(\bar{x}, x)+O\left(d^{2}(\bar{x}, x)\right), \quad(\bar{x} \rightarrow x)$, so the bound in (7) does not differ significantly from the bound (6).

Proof of Theorem 2. Since the theorem is symmetric with respect to $\bar{x}$ and $x$, it suffices to consider the case $|x| \geqslant|\bar{x}|$, in which $d(\bar{x}, x)=|\bar{x} / x-1|$. Let us denote $\bar{x} / x-1=\theta$. Obviously $|\theta|=d(\bar{x}, x) \leqslant \delta<1$. Now

$$
\begin{aligned}
d\left(\bar{x}^{k}, x^{k}\right) & =\left|\bar{x}^{k}-x^{k}\right| / \max \left\{\left|\bar{x}^{k}\right|,\left|x^{k}\right|\right\} \leqslant\left|\bar{x}^{k} / x^{k}-1\right| \\
& =\left|\sum_{n=1}^{\infty} \frac{k(k-1) \cdots(k-n+1)}{n !} \theta^{n}\right| \\
& \leqslant \sum_{n=1}^{\infty} \frac{|k \theta|}{1} \cdot \frac{(|k|+1)|\theta|}{2} \cdots \frac{(|k|+|n-1|)|\theta|}{n} \\
& \leqslant|k \theta| \sum_{n=1}^{\infty} \delta^{n-1}=|k| d(\bar{x}, x) /(1-\delta) .
\end{aligned}
$$


Remark. If $d(\cdot, \cdot)$ is replaced by $|\rho(\cdot, \cdot)|$ in the last theorem, it still remains true. The proof is similar.

Finally let us discuss bounds for the error in sum and difference of approximate quantities and also a relative distance analogue of the conversion from real $\mathrm{rp}$ to complex rp, discussed in [3].

The following theorem is related to the first of these problems and provides bounds for both sum and difference:

TheOREM 3. Let $\bar{x}^{(i)}, x^{(i)}(i=1, \ldots, n)$ be either real or complex numbers and let $d(\cdot, \cdot)$ be defined by (5). If $d\left(\bar{x}^{(i)}, x^{(i)}\right) \leqslant \varepsilon<1(i=1, \ldots, n)$ and $\sum_{j=1}^{n} \bar{x}^{(j)} \neq 0$, then

$$
d\left(\sum_{i=1}^{n} \bar{x}^{(i)}, \sum_{i=1}^{n} x^{(i)}\right) \leqslant \sum_{i=1}^{n} \bar{p}_{i} \frac{\varepsilon_{i}}{1-\varepsilon_{i}} \leqslant \bar{p} \frac{\varepsilon}{1-\varepsilon},
$$

where

$$
\begin{gathered}
\bar{p}_{i}=\left|\bar{x}^{(i)}\right| /\left|\sum_{j=1}^{n} \bar{x}^{(j)}\right|, \quad \varepsilon_{i}=d\left(\bar{x}^{(i)}, x^{(i)}\right) \quad(i=1, \ldots, n), \\
\bar{p}=\sum_{i=1}^{n}\left|\bar{x}^{(i)}\right| /\left|\sum_{j=1}^{n} \bar{x}^{(j)}\right| .
\end{gathered}
$$

As for the second problem, the following is an easy-to-prove result:

TheOREM 4. Let $\bar{x}=\bar{x}_{1}+i \bar{x}_{2}, x=x_{1}+i x_{2}$, where $i=\sqrt{-1}$ and $\bar{x}_{1}, \bar{x}_{2}, x_{1}, x_{2}$ are real numbers, and let $d(\cdot, \cdot)$ be defined by (5). If $\left(\bar{x}_{1}, x_{1}\right) \leqslant \varepsilon, d\left(\bar{x}_{2}, x_{2}\right) \leqslant \varepsilon, 0<\varepsilon<1$, then $d(\bar{x}, x) \leqslant \varepsilon /(1-\varepsilon)$.

Proofs of Theorems 3, 4. These theorems may be easily generalized with no significant changes in the proofs. We refer to the next section for proofs of the generalizations; see Theorems $3^{\circ}, 4^{\circ}$.

3. Relative Distance in Linear Normed Spaces. The definition (5) of relative distance may be easily generalized to linear normed spaces. For $\bar{x}, x$, which are points of such a space, let us define

$$
d(\bar{x}, x)=\|\bar{x}-x\| / \max \{\|\bar{x}\|,\|x\|\}, \quad d(0,0)=0
$$

with $\|\cdot\|$ being the norm in the space.

Error analysis based on this generalization obviously satisfies analogues of (I), (II), and (III); see Section 2. As for (VI) the following is a generalization of Theorem 1:

THEOREM $1^{\circ}$. Let $S$ be a linear normed space with the norm denoted $\|\cdot\|$. If $\|\cdot\|$ originates from an inner product, i.e., if $\|x\|^{2}=(x, x)$, where $(\cdot, \cdot)$ is an inner product in $S$, then $d(\cdot, \cdot)$, defined by $(8)$, is a metric on $S$.

It should be noted that this theorem is sufficiently general to imply that $d(\cdot, \cdot)$, defined by (8), is a metric in Euclidian $n$-spaces, in matrix spaces with the Frobenius norm (see [5]), and in function spaces with the $L_{2}$ norm. So in each of these spaces error analysis, based on (8), will satisfy an analogue of (VI). Note also that Theorem 1 is easily deducible from Theorem $1^{\circ}$. 
Proof of Theorem $1^{\circ}$. Of all the properties of a metric the triangle axiom is the only one which is not self-evident. Let then $x, y, z$ denote three points in $S$, and assume that $\|z\| \geqslant\|y\| \geqslant\|x\|>0$ (the case $\|x\|=0$, which was omitted, is simple). The following three inequalities need to be proved:

$$
\begin{aligned}
d(x, z) & \leqslant d(x, y)+d(y, z) \Leftrightarrow\|x-z\| /\|z\| \\
& \leqslant\|x-y\| /\|y\|+\|y-z\| /\|z\|, \\
d(y, x) & \leqslant d(y, z)+d(z, x) \Leftrightarrow\|y-x\| /\|y\| \\
& \leqslant\|y-z\| /\|z\|+\|z-x\| /\|z\|, \\
d(z, y) & \leqslant d(z, x)+d(x, y) \Leftrightarrow\|z-y\| /\|z\| \\
& \leqslant\|z-x\| /\|z\|+\|x-y\| /\|y\| .
\end{aligned}
$$

The proof of $(\mathrm{A})$ is not difficult:

$$
\begin{aligned}
\|x-z\| & =\|(x-y)+(y-z)\| \leqslant\|x-y\|+\|y-z\| \Rightarrow\|x-z\| /\|z\| \\
& \leqslant\|x-y\| /\|z\|+\|y-z\| /\|z\| \leqslant\|x-y\| /\|y\|+\|y-z\| /\|z\| .
\end{aligned}
$$

The proof of $(\mathrm{C})$ is much the same. In order to prove (B) the following, which is true for norms that originate from inner products, is used:

$$
\alpha, \beta \text { real, }\|u\|=\|v\| \Rightarrow\|\alpha u-\beta v\|=\|\beta u-\alpha v\| .
$$

In order to prove it, notice that

$$
\|\alpha u-\beta v\|=\|\beta u-\alpha v\| \Leftrightarrow(\alpha u-\beta v, \alpha u-\beta v)=(\beta u-\alpha v, \beta u-\alpha v),
$$

and this follows immediately from the bilinearity of inner products. Turning back to (B) one sees that it is equivalent to

$$
\left\|\hat{y}-r_{1} \hat{x}\right\| \leqslant\left\|r_{2} \hat{y}-\hat{z}\right\|+\left\|\hat{z}-r_{1} r_{2} \hat{x}\right\|
$$

where

$$
\hat{x}=x /\|x\|, \quad \hat{y}=y /\|y\|, \quad \hat{z}=z /\|z\|, \quad r_{1}=\|x\| /\|y\|, \quad r_{2}=\|y\| /\|z\| .
$$

This in turn is equivalent to (see (9))

$$
\left\|r_{1} \hat{y}-\hat{x}\right\| \leqslant\left\|\hat{y}-r_{2} \hat{z}\right\|+\left\|r_{1} r_{2} \hat{z}-\hat{x}\right\|=\frac{1}{r_{1}}\left\|r_{1} \hat{y}-r_{1} r_{2} \hat{z}\right\|+\left\|r_{1} r_{2} \hat{z}-\hat{x}\right\|,
$$

which follows immediately from the triangle inequality for the norm because $1 / r_{1} \geqslant 1$.

Powers, products, and ratios are not defined in general linear normed spaces. Therefore one does not expect to find analogues of (IV) and (V) for the generalized $d(\cdot, \cdot)$. There is, however, one exception: Let $\bar{\xi}, \xi$ be scalars (real or complex) and $\bar{y}, y$ points in a linear normed space. Then

$$
d(\bar{\xi} \bar{y}, \xi y) \leqslant d(\bar{\xi} \bar{y}, \xi \bar{y})+d(\xi \bar{y}, \xi y)=d(\bar{\xi}, \xi)+d(\bar{y}, y) .
$$

Finally, let us present generalized versions of Theorems 3 and 4:

THEOREM $3^{\circ}$. Let $\bar{x}^{(i)}, x^{(i)}(i=1, \ldots, n)$ be points in a linear normed space, and let $d(\cdot, \cdot)$ be defined by (8). If $d\left(\bar{x}^{(i)}, x^{(i)}\right) \leqslant \varepsilon<1(i=1, \ldots, n)$ and $\sum_{j=1}^{n} \bar{x}^{(j)} \neq 0$, then

$$
d\left(\sum_{i=1}^{n} \bar{x}^{(i)}, \sum_{i=1}^{n} x^{(i)}\right) \leqslant \sum_{i=1}^{n} \bar{p}_{i} \frac{\varepsilon_{i}}{1-\varepsilon_{i}} \leqslant \bar{p} \frac{\varepsilon}{1-\varepsilon},
$$


where

$$
\bar{p}_{i}=\left\|\bar{x}^{(i)}\right\| /\left\|\sum_{j=1}^{n} \bar{x}^{(j)}\right\|, \quad \varepsilon_{i}=d\left(\bar{x}^{(i)}, x^{(i)}\right), \quad \bar{p}=\sum_{i=1}^{n}\left\|\bar{x}^{(i)}\right\| /\left\|\sum_{j=1}^{n} \bar{x}^{(j)}\right\| .
$$

THEOREM $4^{\circ}$. Let $\bar{x}=\left(\bar{x}_{1}, \ldots, \bar{x}_{n}\right), x=\left(x_{1}, \ldots, x_{n}\right)$, where $\bar{x}_{i}, x_{i}$ are real numbers, and let $d(\cdot, \cdot)$ be defined by (5) for numbers and by (8), where $\|\cdot\|$ is the Euclidian norm, for n-vectors. If $d\left(\bar{x}_{i}, x_{i}\right) \leqslant \varepsilon<1(i=1, \ldots, n)$, then $d(\bar{x}, x) \leqslant \varepsilon /(1-\varepsilon)$.

Proof of Theorem $3^{\circ}$.

$$
\begin{aligned}
d\left(\sum_{i=1}^{n} \bar{x}^{(i)}, \sum_{i=1}^{n} x^{(i)}\right) & \leqslant\left\|\sum_{i=1}^{n}\left(\bar{x}^{(i)}-x^{(i)}\right)\right\| /\left\|\sum_{j=1}^{n} \bar{x}^{(j)}\right\| \\
& \leqslant \sum_{i=1}^{n} \bar{p}_{i}\left\|\bar{x}^{(i)}-x^{(i)}\right\| /\left\|\bar{x}^{(i)}\right\| .
\end{aligned}
$$

Let us denote $\left\|\bar{x}^{(i)}-x^{(i)}\right\| /\left\|\bar{x}^{(i)}\right\|=\rho$, and observe that $\max \left\{\left\|\bar{x}^{(i)}\right\|,\left\|x^{(i)}\right\|\right\} \leqslant$ $\left\|\bar{x}^{(i)}\right\|+\left\|x^{(i)}-\bar{x}^{(i)}\right\|$. It follows that

$d\left(\bar{x}^{(i)}, x^{(i)}\right) \geqslant \frac{\left\|\bar{x}^{(i)}-x^{(i)}\right\|}{\left\|\bar{x}^{(i)}\right\|+\left\|\bar{x}^{(i)}-x^{(i)}\right\|}=\frac{\rho}{1+\rho} \Rightarrow \rho \leqslant d\left(\bar{x}^{(i)}, x^{(i)}\right) /\left[1-d\left(\bar{x}^{(i)}, x^{(i)}\right)\right]$, and the desired inequality follows immediately.

Proof of Theorem $4^{\circ}$. Denote $d\left(\bar{x}_{i}, x_{i}\right)=\varepsilon_{i}$. Then either $\bar{x}_{i}=x_{i}\left(1-\varepsilon_{i}\right)$ (if $\left|\bar{x}_{i}\right| \leqslant$ $\left.\left|x_{i}\right|\right)$ or $\bar{x}_{i}=x_{i} /\left(1-\varepsilon_{i}\right)$ (if $\left.\left|\bar{x}_{i}\right| \geqslant\left|x_{i}\right|\right)$. Hence either $\bar{x}_{i}-x_{i}=-\varepsilon_{i} x_{i}$ or $\bar{x}_{i}-x_{i}$ $=x_{i} \varepsilon_{i} /\left(1-\varepsilon_{i}\right)$. In both cases $\left|\bar{x}_{i}-x_{i}\right| \leqslant\left|x_{i}\right| \varepsilon_{i} /\left(1-\varepsilon_{i}\right) \leqslant\left|x_{i}\right| \varepsilon /(1-\varepsilon)$, so $\|\bar{x}-x\| \leqslant\|x \varepsilon /(1-\varepsilon)\|=\|x\| \varepsilon /(1-\varepsilon)$, and therefore $d(\bar{x}, x) \leqslant\|\bar{x}-x\| /\|x\|$ $\leqslant \varepsilon /(1-\varepsilon)$.

Remark. The bound $\varepsilon /(1-\varepsilon)$, given in Theorem $4^{\circ}$, is not the best possible. It can be shown that for every $0<\varepsilon<1$ and every $n \geqslant 2$ the best bound is $\varepsilon / \sqrt{1-\varepsilon+\frac{1}{2} \varepsilon^{2}} \leqslant \varepsilon /\left(1-\frac{1}{2} \varepsilon\right) \leqslant \varepsilon(1+\varepsilon)$.

A Remark on Some Other Generalizations. $d(\cdot, \cdot)$ may be generalized to function, vector, and matrix spaces also in a different manner. Let $\bar{x}=\bar{x}(t), x=x(t)$ be points of a function space $S$, and assume that $d(\cdot, \cdot)$ is a metric on the union of the ranges of all functions in $S$. A metric on $S$ may be produced by taking appropriate norm of the real valued function $d(t)=d(\bar{x}(t), x(t))$. Thus, e.g., the space of real $n$-vectors may be interpreted as the space of real functions on the finite set $\{1, \ldots, n\}$. Denoting by $\bar{x}=\left(\bar{x}_{1}, \ldots, \bar{x}_{n}\right), x=\left(x_{1}, \ldots, x_{n}\right)$ two points in this space, each of the following is a metric:

$$
\sum_{i=1}^{n} d\left(\bar{x}_{i}, x_{i}\right), \sqrt{\sum_{i=1}^{n} d^{2}\left(\bar{x}_{i}, x_{i}\right)}, \quad \max _{i} d\left(\bar{x}_{i}, x_{i}\right)
$$

These distance measures are insensitive to changes in the scaling of individual vector components. An important difference between them and $d(\bar{x}, x)$ is demonstrated by the vectors $\bar{x}=(1, \varepsilon), x=\left(1, \varepsilon^{2}\right)$ where $\varepsilon$ is small. They will be considered close to each other if distance is measured by $d(\bar{x}, x)$ and significantly separated from each other if distance is measured by any of the last measures.

IBM Israel Scientific Center

Technion City

Haifa 32000, Israel 
1. Vijay B. Aggarwal \& James W. Burgmeier, "A roundoff error model with applications to arithmetic expressions," SIAM J. Comput., v. 8, 1979, pp. 60-72.

2. A. N. Kolmogorov \& S. C. Fomin, Elements of the Theory of Functions and Functional Analysis, Graylock Press, Rochester, New York, 1957.

3. F. W. J. OlVER, “A new approach to error arithmetic," SIAM J. Numer. Anal., v. 15, 1978, pp. 368-393.

4. Pat H. Sterbenz, Floating Point Computation, Prentice-Hall, Englewood Cliffs, N. J., 1974.

5. G. W. Stewart, Introduction to Matrix Computations, Academic Press, New York and London, 1973.

6. J. H. Wilkinson, Rounding Errors in Algebraic Processes, Prentice-Hall, Englewood Cliffs, N. J., 1963. 